Olivier Chassany

\title{
Should European Independent Ethics Committees be dismantled?
}

Received: 28 January 2009

Accepted: 28 January 2009

Published online: 12 February 2009

(C) The Author(s) 2009. This article is published with open access at Springerlink.com

This editorial refers to the articles available at: doi: 10.1007/s00134-008-1343-x and 10.1007/s00134-009-1434-3.

Clinical Research and Development Department is acting as an institutional sponsor for the Assistance Publique-Hôpitaux de Paris. It currently sponsors or manages more than 400 studies (website: http://www.drrc.aphp.fr/).

\section{O. Chassany}

Clinical Research Department, AP-HP and University

Denis-Diderot, Paris, France

\section{O. Chassany $(\bowtie)$}

Département de la Recherche Clinique et du Développement, Assistance Publique-Hôpitaux de Paris (AP-HP),

Hôpital Saint-Louis, 1 avenue Claude Vellefaux,

75010 Paris, France

e-mail: olivier.chassany@sls.aphp.fr

Tel.: +33-1-44841777

Fax: +33-1-44841788

This issue of Intensive Care Medicine presents two aspects of the difficulties that face Independent Ethics Committees (IEC) regarding their role and responsibilities, and not resolved either by the European Directive or by its translation in national laws: a negative point of view on the utility of European IECs through a survey among authors of clinical trials published in some major journals in 2007 [1], and a constructive initiative of the Vienna university IEC, on reviewing interventional studies at minimal risk through an expedited process [2].
Since the release of the 2001 European Union Clinical Trials Directive (EU CTD) (2001/20/EC), an approval from an IEC is mandatory before starting an interventional clinical trial. Moreover, the composition of the IECs has been widened to other categories of the civil society among which of the users' and patients' representatives.

However, the EU Directive is still not entirely enforced by all national countries such as the requirement that explicitly requests a single IEC approval within each national country. In Germany, a local IEC approval is needed for every investigational centre, plus a national IEC opinion, meaning that if you plan a multicenter trial, involving, e.g., 20 German centres, 20 local IEC positive approvals are required [3]. A question comes inevitably from this observation: what is the added benefit of 20 IEC opinions? At least, every supplementary local IEC opinion should improve a little bit the ethical and scientific integrity of the submitted clinical trial. If not and it is probably the case, then the associated-burden appears useless.

The paper of Pehboeck et al. [1] reports the dissatisfaction of 193 authors regarding IECs and clinical trials registers and who have published results of clinical trials in selected major journals in 2007. The negative perception of authors (on a scale from -10 to +10 ) is consistent across all five items in terms of support provided by IEC, effort needed to obtain approval, and friendliness of the approval procedure. The worst negative scores (reaching -4) were for the two items "effort needed to obtain IEC approval caused us to make progress in ethics or to make scientific progress". However, this online survey of corresponding authors which was deliberately brief in order to achieve a high return rate $(80 \%)$ does not permit to give the definite reasons of this high dissatisfaction. It can be a very subjective perception that the IEC submission process is just an additional bureaucratic burden. This dissatisfaction may also reveal a deeper pitfall, i.e. the added value of IECs and their comments on ethics and on methodology of the submitted clinical trials are truly 
questionable. It would have been also interesting to know whether there is a cluster of IECs associated with the worst perception, and to look for the reasons: a skilled and in-depth expertise of the dossier by the IEC (thus useful, although annoying for the applicants) or a useless pernickety review on details. Indeed - and it is a hypocrisy not to face this reality - applicants know quite well which IECs provide balanced and reasonable queries. This last point is one of the major reasons why applicants repeatedly deplore the heterogeneity of IECs and ask for changes [4].

The paper of Pehboeck et al. [1] has the merit to raise the crucial question whether IECs are competent for giving ethical and scientific opinions. Indeed, the role of IECs is not to refine continuously the major ethical principles that rule the clinical research. This is devoted to international organizations (such as Helsinki). For example, it is not the role of IECs to decide, and to have opposite position between IECs, whether in general a placebo arm in clinical research is ethical or not (obviously a placebo arm is unethical when an efficient treatment is available for a fatal or severe pathology). The right inquiry of an IEC for a given clinical trial is to ensure that all actions to reduce the potential risks of being under placebo have been implemented in the protocol (e.g. strict eligibility criteria, frequent visits, add-on therapy, early stopping rules, rescue treatment, unbalanced randomisation scheme...) [5].

The EU directive is considered to impose unnecessary administrative burden and costs which are especially problematic for investigator-initiated and academicsponsored clinical research.

Indeed, EU CTD has been mainly set up for pharmaceutical industry-sponsored clinical trials on medicinal products and thus excludes a broad range of clinical research (e.g. interventional trials of surgical procedures or medical devices). Especially, the EU CTD ignores totally the case of clinical trials at no or minimal risk (added by the research). These latter trials include typically (1) studies with additional exams such as blood puncture, radiography...; (2) evaluation of usual care or of optimised management based on consensus conferences or recommendations, which may not be currently implemented in clinical practice [6]; (3) interventional studies at minimal risk, especially those in primary care and borderline with epidemiology, such as wearing a mask in prevention of flu. However, this type of research currently falls under the same biomedical research regulation as for a new drug clinical trial. This inappropriate burden on minimal risk research is shared across all Europe. Recently, authors reported that in UK, moreover an opinion from a Patient Information Advisory group is also required, which ability to answer quickly may not be its major matter. Authors plead wisely for returning Patient Information Advisory group responsibilities to IECs [6].
The paper by Wolzt et al. [2] presents the initiative of the Vienna IEC, to shorten the review process of clinical trials in which the intervention is at minimal risk. Authors mean by minimal risk, that harm or discomfort anticipated in the research or by standard tests should not be greater than that encountered in daily practice or during the performance of non-invasive routine examinations [2]. The expedited review applies also to medical research projects involving drugs or medical devices when they are used in accordance with their marketing authorisation. Protocols at minimal risk are identified by the chair and reviewed by a selected group of IEC members. The review of this expedited board is then submitted to the full IEC.

This comprehensive attitude of the Vienna IEC, while probably a little outside the current European legislation is a light of hope in a grey horizon, where the nervousness of IECs to assume their responsibilities is equal to their over-legalism (nothing less than, but sometimes more than what is required by the law) and prevents any consideration to help and facilitate the clinical research notably that at minimal or no risk. This experience, fruit of a reasoned and enlightened approach, which allows adapting the extent of the IEC expertise to the expected risks of the study strongly deserves to be known by clinicians and IECs.

\section{Role and responsibilities of IECs should be refined}

IECs should consider whether they do only and respect strictly what has been fixed by the law, knowing that the current law is incomplete and written for industry-sponsored clinical trials with medicinal products. In that case, at least they should not require from applicants and investigators more that what is required by the law [4]. This legalist behaviour, although the most respectful of the current regulation, underestimate a whole part of the clinical research mostly supported by academic and funded in many cases by governmental agencies.

The other solution is to function like an US Institutional Review Board which gives opinion on all clinical studies. This needs of course to endorse some borderline decisions and to accept to tailor the depth of the review process based on the expected risks/constraints of the study. This would allow to quickly review interventional studies with no or minimal risk, and to review even more quickly non-interventional studies. The paper of Wolzt et al. [2] reminds us that since 1998 in USA, Ethics Committees are enabled to give such a fast track approval of clinical studies with minimal risk, including studies of drugs and medical devices. Why what is done since 10 years in USA, could not be implemented and improved in Europe? If nothing changes, there is a risk of having two categories of European IECs: those 
functioning as defined by the current law and those functioning already more as IRBs (some even being agreed by the US administration). The latter may be better predisposed or open-minded to review all types of clinical research and may have an improved assessment of the benefit/risk ratio, which is the basis of the IEC approval. Indeed, open-minded does not mean a less rigorous or less independent process.

The EU Directive should endorse a simplified procedure for minimal risk interventional trials, and should consider that all research on human, including noninterventional studies should be submitted to an IEC, as currently most of authors of these studies seek an opinion from an IRB for them to publish their results. It is worthwhile to mention that the French parliament has just amended the law on biomedical research in that way [7]. Obviously, the extension of a mandatory IEC approval for non-interventional studies is possible only if all IECs do not have an a priori against this type of research. Indeed for some of IECs a non-interventional study is some kind of under-research. If this prerequisite is not satisfied, the risk is high that some IECs will reclassify these studies as interventional with the entire associated useless burden, e.g. as soon as the smallest additional clinician- or patient-reported questionnaire would be part of the outcomes, as it is the case in most studies.

\section{Conclusion}

Haven't we been too far? i.e. too many procedures and safeguards applied without discernment to all kinds of research did kill the original purpose of regulation, that was to protect patients and to ensure the fulfilment of the basic ethical principles [4]. The two papers of this journal issue report accurately two contrasted visages of IECs. However, procedures, role, and expertise of IECs have to be refined rapidly and subsequently applied by all European IECs. The major issue is whether Europe is capable to adjust the administrative burden to the expected risks/ constraints added by the research. If not, some voices may raise with legitimacy for shutting down IECs, and for recreating new well-ordered structures with well-defined roles.

\section{Conflict of interest statement None.}

Open Access This article is distributed under the terms of the Creative Commons Attribution Noncommercial License which permits any noncommercial use, distribution, and reproduction in any medium, provided the original author(s) and source are credited.

\section{References}

1. Pehboeck D, Hohlrieder M, Wenzel V, Benzer A (2009) Submission of clinical studies to Ethics Committees or clinical trials registers - the authors' point of view. Intensive Care Med. doi: 10.1007/s00134-009-1434-3

2. Wolzt M, Druml C, Leitner D, Singer EA (2009) Protocols in expedited reviewtackling the workload of Ethics Committees. Intensive Care Med. doi: 10.1007/s00134-008-1343-x

3. Lienhart HG, Lindner KH, Wenzel V (2008) Developing alternative strategies for the treatment of traumatic haemorrhagic shock. Curr Opin Crit Care 14:247-253
4. Stewart PM (2008) Regulation-the real threat to clinical research. BMJ 337:1085-1087

5. Chassany O, Duracinsky M (1999) Ethics and clinical trials. Fundam Clin Pharmacol 13:437-444

6. Metcalfe C, Martin EM, Noble S, Lane A, Hamdy FC, Donovan JL (2008) Low risk research using routinely collected identifiable health information without informed consent: encounters with the patient information advisory group. J Med Ethics 34:37-40
7. Proposition de loi relative aux recherches sur la personne. Text no 229 voted by the French Parliament on 22 January 2009. http://www.assemblee-nationale.fr/13/ dossiers/recherches_sur_la_personne.asp (accessed on 27 January 2009) 